\title{
6th Annual Meeting of the Complex Trait Consortium
}

\author{
Klaus Schughart · Gary Churchill
}

Received: 25 July 2007/Accepted: 3 August 2007/Published online: 29 September 2007

(C) The Author(s) 2007

The sixth annual meeting of the Complex Trait Consortium took place May 26-29, 2007, in Braunschweig, Germany. The meeting was hosted by the Helmholtz Centre for Infection Research and touched upon a wider variety of topics and organisms than past CTC meetings. The meeting featured a report on the status of the collaborative cross, many excellent talks, panel discussions, outstanding posters, lively discussion, and an entertaining refresher course in basic science by "Professor Singh."

\section{Some highlights ...}

The collaborative cross

An opening panel discussion described progress in breeding of the collaborative cross recombinant inbred lines (CC-RILs). It is exciting to see the rapid progress of this project with three centers reporting on more than 900 existing lines, many of which having reached the G6-G9 generations of inbreeding.

At ORNL, over 400 lines of the CC have now reached at least stage G2:F4 (4 generations of inbreeding), with 386 at $\mathrm{G} 2: \mathrm{F} 5,320$ at G2:F6, 171 at G2:F7, 53 at G2:F8, and 12 at G2:F9. A second wave of 400 new lines has been initiated. ORNL is also setting up a phenotyping center in which all

\footnotetext{
K. Schughart $(\square)$

Department of Experimental Mouse Genetics, Helmholtz Centre for Infection Research and University of Veterinary Medicine Hannover, Inhoffenstr. 7, D-38124 Braunschweig, Germany e-mail: kls@helmholtz-hzi.de

G. Churchill

The Jackson Laboratory, 600 Main Street, Bar Harbor, Maine 04609, USA
}

parents are typed. These tests include wildness scoring, sleep, anxiety, and nociception as well as tissue collection and DNA isolation. Plans are underway to genotype the G2:F8 generation.

Another 139 UK lines have been successfully relocated from Kenya to Tel Aviv. Approximately 400 additional lines are being bred in Perth, Australia. The sizes of the litters are declining (as expected) proportionally to the level of inbreeding, but remain consistent with levels observed in other inbred mouse strains.

Bob Hitzemann reported on the outbred CC-HS population that is breeding at OHSU. He raised concerns about the suitability of standard behavioral testing and suggested that new testing paradigms should be investigated. Coffee break discussions lead to a plan for SNP selection and genotyping of nascent CC-RILs and the CC-HS animals.

A collaborative heterogeneous stock

Jonathan Flint proposed the development of a large-scale heterogeneous stock (HS) population as a sponsored CTC project. The value of large outbred populations has been demonstrated but effective population size and unknown composition of founders have presented some challenges. Subsequent discussions led to the proposal to launch CC-HS breeding from the G2:F1 generation of CC-RILs. G2:F1 is the first generation to carry all eight genotypes and is prior to inbreeding. It was generally agreed that the CC-HS would be a useful and complementary resource population.

Modeling and databases

A systems biology approach to modeling T-cell signaling was presented by Burkhart Schraven. Talks included a 
presentation on the initiation of the CASIMIR project which aims to unify the many existing mouse informatics resources. Concepts for partially automated development of software infrastructures in systems biology were introduced by Ritsert Jansen.

\section{Microarray technology}

Microarray technology and applications are a perennial favorite at the CTC meeting. This year we were treated to a couple of eye-opening reports on array quality and reproducibility. Daniel Ciobanu demonstrated the impact of polymorphisms on probe-level responses in Affymetrix arrays and showed that extensive false-positive cis-QTLs can occur. Rob Williams presented results obtained with different RILs (e.g. the $\mathrm{B} \times \mathrm{D}$ and $\mathrm{L} \times \mathrm{S}$ RILs), in addition to comparing the performance of the Affymetrix versus Illumina platforms.

\section{Genome analysis}

In the area of genomics, we heard talks about extensive variation in genomic structure and expression among (and within) inbred lines due to L1 transpositions (David Symer). Elissa Chesler described the effects of long-range linkage disequilibrium (LD) on mouse association mapping panels and contrasted them with the LD properties of RI lines. We also learned that variation in micro-RNA expression is present and likely to have functional implications among inbred strains.

Gary Churchill described an analysis of the NIEHS Perlegen/resequencing project that has generated more than 8.5 million SNPs from 15 inbred strains. Despite its impressive size and obvious value, there appears to be a high false-negative discovery rate, and it is estimated that as many as 45 million SNPs are present in these strains. The classical strains appear to be largely Mus musculus domesticus (90\% or more) in origin. There are large regions of the genome that are identical by descent across the classical strains and most of the genome has intermediate levels of variation of intrasubspecific origin. Inclusion of the four wild-derived inbred strains contributes substantial variation to the panel ( $75 \%$ of the total). However, the wild-derived strains also carry large regions of introgression from $M . m$. domesticus that may have arisen in the laboratory.

\section{Discovery of QTLs}

It was exciting to hear from many of the talks on quantitative trait loci (QTLs), the bread and butter of the CTC, that many investigators were able to identify genes and mechanisms. Quantitative trait gene (QTG) identification was facilitated by informatics resources, haplotype analysis, and expression QTL. Henning Wittenburg described the role of two linked genes, $A B C G 5$ and $A B C G 8$, that together underlie a gallstone susceptibility QTL and was able to describe a plausible mechanism for the observed pleiotropic effects of this QTL on HDL cholesterol. There were reports of QTLs associated with portions of the genome that are often overlooked, e.g. chromosome $\mathrm{Y}$ and the mitochondrial genomic DNA. Other presentations featured QTLs for kidney disease, brain morphology, and morphometric traits.

QTL studies of tumor susceptibility demonstrated complexity that included imprinting and context dependence. The importance of priming the system in the case of cancer studies was emphasized, providing an important point of intersection between the complex trait and singlegene knockout experimental approaches.

In a refreshing departure from rodent QTL studies, we were taken to a complex trait analysis that utilized the full power of yeast genetics. Using a deletion-complementation strategy, Himanshu Sinha dissected a single QTL into three component genes. Two of these genes showed a strong negative epistatic interaction and the third gene was linked in repulsion phase with respect to the main effects of the other two. This demonstration of the power of yeast genetics may have evoked some envy among the rodentoriented audience, but it was an outstanding illustration of the complexity that can be hidden in a single, apparently additive QTL. Perhaps we need to be a bit more cautious when deciding that we have identified "the" QTG.

Outreach to the scientific community: gene-trap consortia and human genetics

Several participants at this year's meeting represented the gene-trap and mutagenesis communities. A panel discussion focused on the importance of unity in the model organism community as a whole. The complementarity of the single gene and complex traits was illustrated by Karlyne Reilly's cancer QTL experiments that require priming with loss-of-function in tumor suppressor genes and by John French's experiments crossing knockouts onto multiple genetic backgrounds using F1s for toxicology studies.

The importance of studies of natural variation and complexity has been a consistent theme of the CTC that can be enhanced by the power of transgenic technologies. The need for unity within the community and to communicate our ideas more effectively to the public and to government officials was raised.

Jonathan Flint gave an overview on whole-genome association studies in mouse and human. The comparison 
made a clear point that rodent work is an essential rather than alternative strategy. The analysis of multiple invasive phenotypes to probe the physiology of complex traits, coupled with analysis of gene expression, protein, and metabolite profiles in multiple tissues, is possible only in model organisms. Integrating such information will be essential if we are going to understand how genes operate in complex phenotypes.

The participants of the meeting agreed that a greater emphasis on translation to human disease is needed. We must engage more human geneticists with the work of the CTC group and demonstrate the power and necessity of rodent models to further our understanding of human diseases.

$\ldots$ and some business

The next CTC meeting will take place in Montreal, Canada, in May 2008 and will be organized by Christian Deschepper (christian.deschepper@ircm.qc.ca). In 2009, the meeting is planned to be held in Manchester, UK, organized by Werner Müller (wmu@helmholtz-hzi.de).
There was general agreement that the members of the CTC wish to continue the trend of broadening the scope of organisms and technologies presented at this meeting and of opening the CTC to a community of researchers beyond the rodent.

The majority of the participants strongly felt that the current format should be maintained for future meetings. They were also very appreciative that the CTC is a meeting that is independent of other mammalian genome meetings and provides a forum for interaction and development of new ideas within its community.

The 2007 meeting of the CTC was engaging and stimulating with frank discussions and the spawning of new ideas. More than one attendee claimed it was one of the best meetings they had ever attended. For this the community owes thanks to the HZI for hosting the meeting, to the organizing committee, and especially to the local organizing team for setting up a relaxed atmosphere that enabled (most of) us to make it through the $3+$ days of intense and sometimes humorous activities.

... And always remember: "Don't forget the buffer." (Prof. Singh) 